\title{
Trois types d'autorité pour trois modes de relation pédagogique
}

\section{Christian Reynaud}

\section{OpenEdition}

\section{Journals}

Édition électronique

URL : http://journals.openedition.org/trema/516

DOI : 10.4000/trema.516

ISSN : 2107-0997

\section{Éditeur}

Faculté d'Éducation de l'université de Montpellier

\section{Édition imprimée}

Date de publication : 1 mars 2007

Pagination : 69-80

ISSN : 1167-315X

\section{Référence électronique}

Christian Reynaud, «Trois types d'autorité pour trois modes de relation pédagogique », Tréma [En ligne], 27 | 2007, mis en ligne le 29 septembre 2010, consulté le 10 décembre 2020. URL : http:// journals.openedition.org/trema/516 ; DOI : https://doi.org/10.4000/trema.516

Ce document a été généré automatiquement le 10 décembre 2020.

Trema 


\title{
Trois types d'autorité pour trois modes de relation pédagogique
}

Christian Reynaud

\author{
I. Introduction
}


1 La réflexion sur l'autorité exposée dans cet article répond à une insatisfaction, voire à un malaise, que j'ai bien souvent ressenti dans l'exercice de mon travail d'enseignant. Cette insatisfaction, ou ce malaise, parfois fugace en cours de séance, ou plus prégnant à la suite d'un enseignement, me paraissait souvent symptomatique d'un écart entre l'objectif que je voulais atteindre et une évaluation (plus ou moins spontanée) de mon action de formation. Ce type d'analyse m'amenait alors généralement à revoir mon évaluation «spontanée » pour considérer que les objectifs avaient bien été atteints;

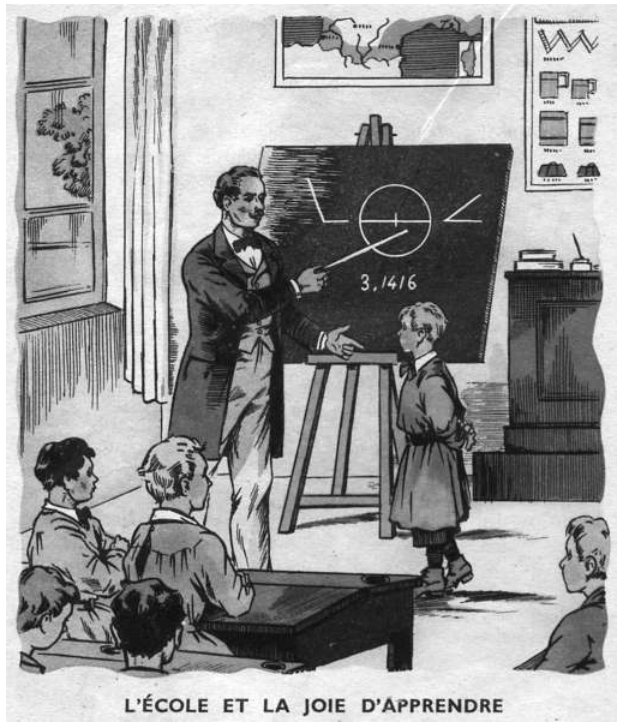
l'insatisfaction ou le malaise était donc injustifié ...

2 Aujourd'hui, c'est la négation de la légitimité du symptôme qui me semble injustifiée.

3 En effet, notre idéal éducatif, surtout depuis quelques décennies, s'est construit en se dissociant de l'autoritarisme qui l'avait longtemps imprégné (GUÉRIN, 2001 ; GUILLOT, 2006). Cette vision nouvelle de l'acte pédagogique, comme une relation visant à favoriser l'accès de l'enfant à son autonomie, me paraît une avancée fondamentale de notre société. Or, j'associe aujourd'hui mon embarras face à un écart supposé entre l'objectif d'enseignement poursuivi et le but réellement atteint, à une impression de manipuler les apprenants. Je développerais alors l'hypothèse que la manipulation serait un mode d'expression de l'autorité de l'enseignant.

4 Je tenterai, à la lumière de diverses approches, une analyse croisée de ce type de situation qui m'amènera notamment à préciser une définition de la manipulation et à la différencier de deux autres modes de relation pédagogique afin de proposer l'identification de trois types d'autorité.

5 L'enseignant, en abordant une séance, a un projet de formation puisqu'il souhaite pouvoir mettre en œuvre les outils d'enseignement qu'il a élaboré dans sa préparation.

6 N'a- t-il pas l'habitude de manipuler les apprenants afin qu'ils se soumettent au dispositif d'enseignement qu'il a prévu?

7 J'ai souvent entendu dire que l'enseignement comprenait obligatoirement une part de manipulation qu'il fallait se résigner à accepter. Cette affirmation ne me satisfait pas car je voudrais travailler sur l'hypothèse inverse, c'est - à - dire que je vais tenter d'apporter des éléments de réponse à la question : peut-on enseigner sans manipuler?

8 En effet, la manipulation pourrait constituer une technique qui, si elle n'est pas incontournable, paraît au moins très efficace pour atteindre les objectifs d'un enseignement. La manipulation constituerait un outil parmi d'autres pour susciter l'intérêt des apprenants et éviter des confrontations désagréables ou la passivité, en imposant une dynamique utilisée pour « le bien commun ». Alors, pourquoi s'en passer? Si je manipule les apprenants, cette action serait justifiée si tout le monde en tirait profit. 
9 Le problème me semble se situer dans la possibilité que la manipulation ne s'adresse pas seulement aux apprenants mais aussi à une dimension masquée de la personnalité des enseignants. En manipulant, le manipulateur pourrait être la première victime de son action en perdant de vue son objectif. Ainsi, en manipulant les apprenants pour qu'ils se soumettent au dispositif d'enseignement prévu, l'enseignant abandonnerait son objectif d'apprentissage. En ce sens, les manipulations des enseignants ne seraient pas aussi délibérées que les techniques comportementales susceptibles d'être utilisées pour manipuler les « honnêtes gens » (JOULE et BEAUVOIS, 1987).

Cette question me semble avoir un corollaire lorsqu'une tâche proposée, un type de démarche utilisé sont parfois perçus comme des manipulations et peuvent être refusés par les apprenants (sous une forme plus ou moins conflictuelle). C'est souvent le cas quand un objectif intermédiaire reste caché pour les apprenants et que l'activité ne semble profiter qu'au bien - être de l'enseignant. Dans ce cas, l'enseignant se retrouve déçu et décontenancé par certaines réactions inattendues. Il peut alors avoir une réaction inadaptée car elle n'engendrera que des frustrations supplémentaires. N'ayant pas identifié ce qui fait problème (la suspicion d'une manipulation), il traitera le conflit par la fuite dans une autre direction, sans espoir de répondre au problème.

11 Comment gérer cette situation autrement, sans créer de frustration?

12 Ma problématique s'insère dans un mouvement social plus large qui me semble se cristalliser dans une hypersensibilité aux phénomènes de manipulation (DELOUVÉE, 2005) : les médias, avec le consentement implicite de leurs publics, cherchent à mettre au grand jour les différentes voies pour maquiller la réalité du fonctionnement de nos institutions - les «affaires ». Ces histoires, parce qu'elles font résonner un sentiment de vulnérabilité, répondent à notre impression d'être à la merci des manipulateurs malfaisants (SOULEZ, 2004). Du côté des défenseurs (avocats et collègues de ceux qui sont accusés), le règne hégémonique de l'efficacité, comme valeur suprême de notre société, est généralement invoqué pour justifier les comportements mis en cause. J'interrogerai donc le rôle des valeurs dans la justification de l'usage de la manipulation comme expression de l'autorité de l'enseignant. Ce problème de valeur touche à des craintes, certainement légitimes, qu'il me paraîtra utile d'analyser car ces peurs pourraient justement constituer des bases pour les phénomènes de manipulation.

13 Ce mot ayant généralement une forte connotation péjorative, il me semble ensuite important de le définir avec précision dans le cadre de ce travail ${ }^{1}$. Pour cela, je le relierai au problème de la motivation, d'une part, et aux supports biologiques des structures cognitives mises en jeu, d'autre part.

\section{Un problème de valeur : coopération contre efficacité}

14 Argumenter sur un problème de valeur pour répondre à ma problématique n'est pas directement évident. J'ai déjà évoqué l'affirmation d'une dimension manipulatrice incontournable dans la relation pédagogique, ce qui rendrait la discussion sans objet. Au travers de cette affirmation, j'entends dire que les enseignants doivent user de la manipulation car il n'y aurait aucune autre façon d'amener les apprenants à agir « pour leur bien ». 

l'enseignement, a été de distinguer une manipulation positive et une autre négative. La manipulation négative ne servant que l'intérêt du manipulateur, sa version positive viserait à conduire le manipulé vers une position plus intéressante pour lui. Dans cette position, un jugement de valeur tend à dispenser d'argumentation sur la nécessité : la manipulation est une «bonne » ou une «mauvaise » chose, une technique acceptable et donc automatiquement acceptée si elle est nécessaire ou, à l'inverse, une négation d'un principe moral.

16 Ces jugements de valeur (agir pour leur bien, manipulation bonne ou mauvaise) m'autorisent toutefois à poser la question éthique : quel est le système de valeur sur lequel s'appuient les jugements et est - il partagé?

La distinction de Jean - Pierre OBIN me semble alors éclairante : il différencie éthique et technique ${ }^{2}$ car " Avec la technique, l'efficacité est maîtresse du jeu et le choix [le jugement] est relativement facile: ce sont les critères d'utilité, de rendement, de rapidité qui s'imposent d'euxmêmes; le résultat l'emporte sur la manière de faire. Avec l'éthique, le choix est toujours difficile, rarement consensuel, souvent controversé » (OBIN, 2006).

18 Avec un système de valeur masqué par les critères de la technicité, il me semble possible de mesurer le résultat d'une manipulation. La manipulation semble donc bien efficace. Mais cette efficacité ne signifie pas que d'autres méthodes n'aboutiraient pas à des résultats analogues.

19 L'aliénation consistant à considérer comme indispensable quelque chose qui se révèle efficace, s'ancre dans la croyance que l'enseignant devrait contrôler la déstabilisation cognitive et affective nécessaire à tout apprentissage, est donc essentiel dans tout processus d'enseignement qui viserait un changement de représentation.

20 Le recours à la manipulation permet peut - être d'atteindre les objectifs de l'enseignant, mais dans le même temps, il renforce une croyance collective qui légitime l'usage de la force dans les dispositifs d'apprentissage. Je suis donc persuadé qu'il est dans l'intérêt de tous que les enseignants n'utilisent pas la manipulation, non parce qu'elle serait « mauvaise », mais parce qu'elle constitue un pouvoir sur autrui. En effet, «L'efficacité est certes une valeur du service public, mais elle vit constamment en tension avec les valeurs politiques et morales qui gouvernent notre société et notre institution» (OBIN, 2006). Pour la manipulation et dans le cadre de l'enseignement, il ne s'agit pas tant de savoir si elle est bonne ou meilleure qu'une autre technique, qu'un autre mode de relation pédagogique, mais plutôt d'expliciter en quoi elle s'oppose à ou justifie des valeurs politiques et morales. Or, il me semble que la manipulation ne peut justifier que le règne du plus fort et l'aliénation des choix personnels, en s'opposant à un partage du pouvoir dans la démocratie et à la défense des libertés individuelles (cela devrait être confirmé lorsque j'aurai défini plus précisément la manipulation).

21 L'usage de la manipulation dans l'enseignement serait donc contestable car il cautionne un modèle d'enseignement proche de la violence en ce sens qu'il constitue un comportement affaiblissant pour autrui (FAVRE et al. 2005).

$22 \mathrm{Au}$ - delà du positionnement éthique, il me semble donc nécessaire d'interroger l'efficacité de la manipulation dans le maintien d'une dynamique de groupe dans la classe. Pour cela je propose d'explorer la piste proposée par Charles ROJZMAN : prendre en compte les angoisses et les peurs de chacun. 


\section{Prendre en compte les peurs engendrées par le contexte social ou les utiliser?}

«Il est rare que je n'entende pas, dans un stage, une phrase comme : « Toi, tu es sincère, mais tu ne te rends pas compte que tu es manipulé. » (ROJZMAN, 1998).

Cette anecdote, rapportée par Charles ROJZMAN, en opposant la sincérité (supposée) du formateur à la duplicité de son autorité supérieure, me semble révélatrice des peurs sur lesquelles peut s'appuyer la manipulation (ici dans le contexte de la formation d'adultes). Cette peur de la duplicité correspond, pour moi, à une peur de l'agression et au - delà une peur de ne pas être aimé. Ces peurs expriment toutefois des besoins : besoin de sécurité et besoin d'amour.

À ces peurs, dans le cas de l'enseignement, pourrait se rajouter la peur de l'inconnu face à des enseignants qui savent et qui distribuent leur savoir à des apprenants. Cette peur correspondrait alors à un besoin de certitude pour se sentir en sécurité (on retrouve le besoin de sécurité).

La manœuvre de la manipulation consisterait alors à faire croire que ces besoins seront satisfaits si la personne manipulée s'engage dans une action dont elle ne connaît pas les effets a priori.

Et c'est bien là que se joue la transaction dans l'enseignement car comment l'enseignant pourrait-il connaître le changement qui va s'opérer chez l'apprenant à travers l'acte d'enseignement? Il peut bien sûr en avoir une idée, mais il ne peut garantir que les besoins des apprenants ne seront pas mis à mal.

Il me semble qu'il y a dans la représentation coutumière de l'autorité de l'enseignant une énorme difficulté que la manipulation permet de surmonter plus ou moins facilement : si le manipulateur est assez habile il arrivera à faire croire aux apprenants qu'il peut garantir un cadre sécurisant et généralement qu'il pourra satisfaire leur besoin d'amour. pourraient être bien plus longs et plus difficiles à mettre en place sans le recours à la manipulation ...

Dans cette hypothèse quelle piste peut-on proposer? Un travail sur les motivations devrait me permettre d'avancer quelques pistes.

\section{Analyser les motivations pour identifier les projets de chacun}

L'enseignant a généralement $d u$ mal à reconnaître des manipulations dans ses comportements. Il les considère comme des techniques pour guider les apprenants vers l'objectif de son enseignement. En toute bonne foi, il cherche ainsi souvent à motiver les apprenants. Mais la question de la motivation fait apparaître que la manipulation a peu de chance d'être satisfaisante. Demandons-nous en effet quel type de motivation est recherché par l'enseignant. Pour poser cette question il faut toutefois disposer d'un modèle qui permet de distinguer plusieurs types de motivation. 

modèles. En définissant une polarité entre origine intrinsèque et extrinsèque de la motivation, cette théorie oppose le besoin d'autonomie à des régulations externes. Ainsi, un apprenant motivé intrinsèquement s'intéresserait aux activités qui lui sont proposées pour elles - mêmes, pour leurs contenus, tandis qu'une activité pratiquée pour ses effets une note, une appréciation positive de l'enseignant - serait dite extrinsèquement motivée. Cependant, cette gradation appliquée à l'enseignement se heurte au paradoxe de l'injonction « soit autonome » adressée à l'apprenant. distinguent trois systèmes de motivation. Le modèle présenté suggère d'abord l'existence de deux systèmes de motivation interdépendants, liés à deux couples de satisfaction / frustration opposés et complémentaires. Dans le premier système (SM1) la gratification (ou l'insatisfaction) est perçue dans les situations de dépendance; dans le second cas (SM2) le plaisir (ou le déplaisir) est associé à une position de responsabilité et à l'autonomie. Ces deux systèmes seraient appelés à se relayer au cours du développement d'un être humain. Le SM1 serait prépondérant au cours des premiers âges de la vie et nous pousserait à rechercher la satisfaction de nos besoins essentiels (biologiques et psychologiques) grâce à l'intervention d'autrui ${ }^{3}$. Le SM2 pourrait se développer progressivement et nous engager à rechercher de nouvelles solutions pour répondre à nos besoins qui nous libèrent de la dépendance ${ }^{4}$. Quel que soit le moment de la vie concerné, la somme des parts de chacun des systèmes est considérée comme constante, c'est - à - dire que la motivation issue du SM1 devrait diminuer au cours de la vie de l'être humain pour être progressivement remplacée par une source de motivation SM2.

Cependant, le développement de ce deuxième système de motivation pourrait être entravé par un parasitage renforçant l'importance du SM1, et faisant exister une troisième source de plaisir / frustration. Dans ce SM1p ${ }^{5}$, la satisfaction serait associée au maintien d'une dépendance anormalement élevée. Il déséquilibrerait le rapport entre les deux autres systèmes de motivation en associant le couple plaisir / frustration à des comportements automatiques, construits le plus souvent pendant l'enfance, comme une réponse d'adaptation à une demande de l'environnement humain (motivation SM1). Parce qu'ils perdurent à l'insu de la conscience, comme des prescriptions ${ }^{6}$ dont la pertinence n'est pas remise en cause, ils s'opposent ensuite à l'épanouissement complet de la personne (au développement du SM2).

Grâce à ce modèle, il devient possible de proposer une définition de la manipulation dans le cadre de mon travail. Manipuler correspondrait à utiliser les vulnérabilités affectives d'autrui à son insu afin d'obtenir qu'il pense ou qu'il agisse pour atteindre l'objectif fixé par le manipulateur, c'est - à - dire que le manipulateur utiliserait les motivations issues du SM1p d'autrui à son profit. Cette définition de la manipulation implique, comme dans la violence (FAVRE et al., 2005), un rapport à l'autre où celui - ci devient un objet utilisé pour satisfaire mes propres besoins.

En contrepartie de cette définition, il me faut préciser quel type d'action permettrait de favoriser le développement de la responsabilité ${ }^{7}$ du sujet, ce que j'appellerais " influencer ». La théorie de l'influence sociale (BUTERA et al. 1998; BUTERA et MUGNY, 2001) suggère que le comportement d'un sujet est conditionné par des normes et des attitudes de groupe ou d'individu. Influencer reviendrait à négocier une démarche et une action favorables aux deux partis que sont l'enseignant et les apprenants (MOSCOVICI, 1994, LAURENS, 2005). Dans le modèle de FAVRE et FAVRE (1993) cela signifierait faire 
appel aux motivations du SM1 qui permet de reconnaître les forces (et les faiblesses) de chacun, sans inhiber l'intervention du SM2 qui permettra l'acquisition d'une autonomie nouvelle.

L'être humain semble avoir développé au cours de son évolution une capacité spécifique à se représenter ses semblables : l'empathie ${ }^{9}$. Elle se perfectionnerait à l'usage au cours de l'enfance à partir d'une faculté innée à se laisser « contaminer » par les émotions et les sentiments de nos proches - et un isolement total d'un enfant dès la naissance entraverait 
son développement aussi sûrement que l'obscurité totale atrophierait les centres de la vue (FAVRE et al. 2005).

La structure cérébrale impliquée semble correspondre aux lobes frontaux dans lesquels :

- d'une part les détails spécifiques décrivant des sujets « autres " peuvent être intégrés et interprétés (s'ils sont endommagés, les actions d'autrui peuvent être perçues et remémorées, mais en perdant leur lien avec la spécificité de la personne qui les effectue);

- et d'autre part une régulation des émotions semble possible (des lésions des lobes frontaux peuvent entraîner des troubles de l'humeur et notamment une moindre tolérance à la frustration).

Les lobes frontaux de l'être humain moderne semblent constituer une évolution de structures commençant à se développer chez les primates et présentes chez les mammifères primitifs. Un des rôles de ces structures, concerne la représentation du temps. «Cet ultime équipement nerveux nous doterait ainsi de la faculté de nous représenter le temps futur dans la mesure où il permet de construire un programme d'actions et de vérifier son exécution. Et à la suite d'observations de lésés et d'handicapés cérébraux frontaux, on peut faire l'hypothèse que les lobes frontaux interviennent fonctionnellement pour nous permettre de nous détacher du temps passé et de ses modes de fonctionnement " (FAVRE, 1993, p. 23). Ces structures seraient alors impliquées dans les processus d'apprentissage en permettant le développement de la capacité à sortir des schémas d'action répétitifs pour expérimenter de nouveaux comportements.

4 Un autre rôle, qui pourrait être crucial dans le développement de l'espèce humaine, pourrait être de contribuer à rendre la manipulation d'autrui possible. En permettant à la fois de se représenter autrui et de s'imaginer son comportement dans le futur, les lobes frontaux nous permettraient d'apprendre comment dissimuler nos propres motivations en comprenant comment les autres nous perçoivent. Si vous savez comment donner l'impression d'être soumis et serviable - quelles que soient vos intentions - vous avez plus de chance de dérober de la nourriture ou d'avoir des rapports sexuels avec la compagne de quelqu'un d'autre (problématique qui pourrait avoir été très importante pour les premiers hominidés). La manipulation pourrait ainsi s'être développée comme une stratégie comportementale permettant de s'adapter au fonctionnement dominants / dominés classique - et donc de continuer à assurer la transmission des gènes.

Mais réussir à se représenter autrui et à prévoir son comportement avec succès peut aussi conduire à reconnaître ce qui serait «bon" pour lui. Le développement de la capacité d'empathie, s'il permet d'améliorer l'efficacité de la manipulation devrait aussi permettre d'obtenir qu'un individu agisse pour son propre bien-être (ce qui correspondrait à de l'influence selon ma proposition). Une logique sous - jacente persiste toutefois dans l'idée que l'autre n'est pas capable de reconnaître ce qui est bien pour lui, qu'il reste dépendant du jugement porté par un autre. Avec le développement de l'empathie, une deuxième logique pourrait venir supplanter la première : en prenant conscience que l'autre n'est pas si différent de moi, qu'il a de «bonnes » raisons de faire ce qu'il fait, de dire ce qu'il dit, de penser ce qu'il pense, je pourrai le considérer comme un alter ego capable de décider de son sort. Il me semble alors facile d'envisager une relation d'accompagnement (selon ma proposition précédente). 


\section{Autorité et relation éducative} collectivement nous choisissons de ne plus nous y soumettre en construisant un modèle de relation basé sur la coopération, alors, l'empathie devient une capacité précieuse, à développer et à mettre au service des valeurs qui nous sont chères.

Il faudrait alors identifier quelles sont les pratiques pédagogiques qui pourraient se traduire par une utilisation optimale des lobes frontaux des apprenants (par rapport à d'autres, qui sont proches de la manipulation et ne semblent pas requérir la présence ou le fonctionnement de la dernière structure nerveuse que nous ayons développée au cours de l'évolution). L'opposition classique entre autorité et laxisme (HOUSSAYE, 2001) amène souvent à rechercher un juste milieu, un pis - aller. A propos de la dynamique des groupes, une référence incontournable dans les ouvrages de sciences de l'éducation, concerne les travaux sur le leadership de LIPPITT et WHITE (1943). Ils définissent trois types de fonctionnement des groupes :

- Leadership autocratique ou autoritaire : les décisions sont prises par un responsable seul, qui reste à l'écart de la vie du groupe.

- Leadership démocratique ou participatif : les décisions résultent des discussions provoquées par un leader et tiennent compte de l'avis du groupe.

- Leadership permissif ou « laissez faire » : un responsable précise les objectifs et les moyens attribués au groupe, mais adopte ensuite un comportement passif. Le groupe jouit d'une totale liberté pour prendre ses décisions.

Les expériences reprenant cette typologie donnent des résultats significatifs: la productivité des groupes à leadership démocratique est nettement supérieure aux autres. À partir de ce constat, «l'autorité démocratique » (TOZZI, 2006) peut devenir un modèle idéal pour l'enseignement.

Toutefois, la relation éducative n'implique pas seulement des prises de décision et elle doit faire appel à toutes les ressources (cognitives et affectives) de celui auquel elle s'adresse. Elle devrait donc s'appuyer alternativement sur l'influence et l'accompagnement, en fonction des besoins de l'apprenant.

\section{Conclusion}

Pour assurer l'éducation de sa descendance, l'homme s'est doté au cours des âges de dispositifs d'enseignement de plus en plus élaborés, améliorant sans cesse l'efficacité de son intervention éducative. Cependant ces dispositifs doivent s'insérer dans une société où l'abondance et la rapidité des évolutions sont des problèmes à gérer.

Dans ce contexte, la manipulation semble une stratégie possible pour atteindre les objectifs d'un enseignement, mais elle n'apparaît pas adaptée à la complexité des relations qui se tissent dans notre société. Elle semble ainsi en contradiction avec l'abandon des valeurs de nos sociétés traditionnelles fondées sur des rapports de 
domination / soumission. Si les valeurs que nous souhaitons afficher (et transmettre) sont plus proches de la coopération, efficace mais respectueuse des spécificités de chacun, il faudra toutefois dépasser l'obstacle consistant à penser que dans toute relation il y a de la manipulation, c'est - à - dire utilisation de l'autre comme un objet. L'enjeu de ce dépassement me semble très important dans la mesure où il contribuerait à montrer que la violence, consistant à affaiblir l'autre en niant sa position de sujet, n'est (peut - être) pas une fatalité.

Pour favoriser ce dépassement, je propose de différencier trois types d'autorité mis en jeu dans les dispositifs pédagogiques.

Ce repérage de trois types d'autorité pourrait non seulement permettre d'utiliser notre capacité empathique envers les autres, mais aussi de la mettre au service d'une meilleure écoute de ce qui se passe en nous afin d'éviter de nous éloigner de nos propres projets.

Le premier type d'autorité, proche de ce que l'on appelle généralement l'autoritarisme, serait identifiable parce qu'il correspondrait à une manipulation de celui auquel l'autorité s'adresse.

61 Le deuxième type serait associé à une relation d'influence qui, bien que nécessaire au développement psychologique, maintiendrait le sujet (apprenant) dans une relation de dépendance par rapport à l'autorité.

Le troisième type d'autorité, en renvoyant chacun à la responsabilité de ses pensées, de ses sentiments et de ses actes, permettrait alors de désamorcer les risques de violence liés à toute tentative de manipulation, et serait associé à un accompagnement responsabilisant.

63 A partir de cette taxonomie, une analyse des situations didactiques me semble possible afin de préciser en quoi elles pourraient être des exemples de non - manipulation (et donc des modèles de non - violence). Ce type d'analyse pourrait être le prix à payer pour concilier les missions d'instruction et d'éducation de l'enseignant.

\section{BIBLIOGRAPHIE}

BUTERA, F. et MUGNY, G. (2001) The use of social influence research. In F. BUTERA and G. MUGNY (Eds), Social influence in social reality. Seatle : Hogrefe \& Huber. P. 1 - 5.

BUTERA, F., GARDAIR, E., MAGGI, J. et MUGNY, G. (1998) Les paradoxes de l'expertise : influence sociale et (in)compétence de soi et d'autrui. In J. BAILLÉ, J. Py \& A. SOMAT (Eds), Psychologie sociale et formation professionnelle : propositions et regards critiques. Rennes : Presses Universitaires de Rennes. P. 109-123.

DELOUVÉE S. (2005) L'image de la psychologie sociale dans les forums de discussion sur l'Internet. Hermès 41. P. 159 - 165.

FAVRE C. et FAVRE D. (1993) Un modèle complexe des motivations humaines. Revue de psychologie de la motivation 16. 
FAVRE D. (1993) Approche neuro - pédagogique des lobes frontaux humains. Les Sciences de l'Éducation 5. P. 23 - 44.

FAVRE D. (1998) Le rôle du langage dans la régulation des comportements violents. Revue Empan 32. P. $41-45$.

FAVRE D. (2001) La dimension affective dans les apprentissages scientifiques. In P. Clément, H - R DAHMANI et F. KHAMMAR (Eds), Didactique de la biologie : recherches, innovations, formations. ENSH - Blida - Algérie. P. 83 - 94.

FAVRE, D., JOLY, J., REYNAUD, C. et SALVADOR, L.L. (2005) Empathie, contagion émotionnelle et coupure par rapport aux émotions. Enfance 2005(4). P. 363 - 382

GUÉRIN, V. (2001) A quoi sert l'autorité. Lyon : Chroniques sociales.

GUILLOT, G. (2006) L'autorité en éducation : sortir de la crise. Paris : ESF.

HOUSSAYE, J. (2001) Autorité ou éducation. Paris : ESF.

JOULE, R. - V. et BEAUVOIS, J. - L. (1987) Petit traité de manipulation à l'usage des honnêtes gens. Grenoble : PUF.

LIPPITT, R. and WHITE, R.K. (1947) An experimental study of leadership and group life. In A. LEVY, Psychologie sociale, textes fondamentaux. Paris : Dunod. P. 278 - 292.

MOSCOVICI, S. (1994) L'influence n'est pas la manipulation - Propos recueillis par Jacques LECOMTE. Sciences Humaines 37. P. 38 - 41 ;

OBIN, J.P. (2006) Technique, éthique et esthétique dans la direction des établissements scolaires. http:// www.esen.education.fr/esentv/obin/index_obin.phtml

PRAIRAT, E. (2005) Autorité et respect en éducation. Le Portique, Le Respect : http://

leportique.revues.org/document562.html

ROGERS, C. (1971) Liberté pour apprendre ? Paris : Dunod.

ROJZMAN, C. (1998) Savoir vivre ensemble. Paris : La découverte.

ROSENBERG, M. (1999) Les mots sont des fenêtres (ou bien ce sont des murs). Paris : Syros.

SOULEZ, G. (2004) Rhétorique, public et « manipulation ». Hermès 38. P 89 - 95

TOZZI, M. (2006) L'autorité démocratique : une provocation conceptuelle ? Les cahiers du CERFEE 21 : http://www.philotozzi.com/articles/article264.htm

\section{NOTES}

1. Il convient d'indiquer qu'une large bibliographie aurait pu fournir une définition plus exhaustive de la manipulation en balisant les études qui lui sont consacrées, mais ce concept a toujours une acception très large et souvent connotée négativement dans son utilisation (à part, peut être, dans des contextes tels que la formation aux techniques de vente !). Il ne m'a donc pas semblé nécessaire, dans le cadre de ce travail, de discuter de définitions construites pour répondre à d'autres problématiques et j'ai préféré en préciser la portée à l'intérieur de ce travail, lorsque la question des motivations me permettra de la faire.

2. Jean - Pierre OBIN propose, en fait, un triptyque pour décoder les «situations les plus délicates à traiter pour les chefs d'établissements » : la logique technique, la dimension éthique et le rôle de l'esthétique (OBIN, 2006). 
3. Les conduites plus ou moins automatiques qu'il produit, seraient déterminées par des références externes à l'individu, c'est - à - dire que les émotions ou les sentiments ressentis seraient associés à une cause extérieur.

4. Sa référence, qui fonde la sécurité du sujet, est interne: elle résulte de l'intériorisation de l'affection et de l'estime reçues des adultes dans le SM1 et repose sur l'estime de soi.

5. L'existence de ce "parasitage» de la motivation peut être retrouvé dans les travaux de « FREUD qui a été l'un des premiers à montrer comment l'expression de la pulsion de vie peut être contrariée au cours du développement et comment son refoulement induit la persistance de désirs infantiles chez l'adulte » (FAVRE et FAVRE, 1993)

6. Ces prescriptions correspondraient à des injonctions ou des recommandations plus ou moins verbalisées et répétées par les parents, les frères et sœurs, la nourrice, les enseignants et parfois même, les médias.

7. Il me faut préciser que, pour moi, l'enseignant doit être, avant tout, un agent de la socialisation des apprenants. L'objectif d'un enseignement devrait alors être une éducation à la citoyenneté, c'est - à - dire un dispositif favorisant le développement de la responsabilité de l'apprenant.

8. Cette relation d'accompagnement ne correspond pas parfaitement à la non - directivité prônée par Carl ROGERS (1971) et ses adeptes car il ne s'agit pas de nier l'importance de la directivité : le processus de développement du SM2 n'est possible qu'en substitution du SM1. La relation d'accompagnement est donc asymétrique car l'accompagnateur devra tenir compte des contraintes associées à l'activation du SM1.

9. Pour définir (de manière approximative et provisoire) l'empathie, je citerais « l'empathie est une façon de comprendre avec respect ce que les autres vivent. Selon le philosophe chinois Tchouang - Tseu, l'empathie véritable exige que l'on écoute de tout son être: "L'écoute exclusivement auditive est une chose. L'écoute intellectuelle en est une autre. Mais l'écoute de l'esprit ne se limite pas à une seule facultél'audition ou la compréhension intellectuelle. Elle requiert en état de vacuité de toutes les facultés. Lorsque cet état est atteint, l'être tout entier est à l'écoute. On parvient alors à saisir directement ce qui est là, devant soi, ce qui ne peut jamais être entendu par l'oreille ou compris par l'esprit » » (ROSENBERG, 1999).

\section{RÉSUMÉS}

Décomposer la motivation humaine en trois systèmes permet de distinguer trois modes de relation pédagogique qui activent différemment ces systèmes: la manipulation, l'influence et l'accompagnement. Dans cette distinction apparaissent les composantes relationnelles de l'autorité et une typologie peut être proposée.

The decomposition of the human motivation into three systems makes it possible to distinguish three teaching relation modes, which activate those systems differently: the manipulation, the influence and the accompaniment. In this distinction appear the relational components of the authority and a typology can be suggested.

\section{INDEX}

Mots-clés : accompagnement, influence, manipulation, motivation

Keywords : accompaniment, influence, manipulation, motivation 


\section{AUTEUR}

\section{CHRISTIAN REYNAUD}

Maître de conférences en Sciences de l'Éducation, IUFM de Montpellier, Équipe d'accueil n³749 (LIRDEF) 